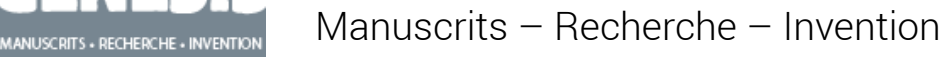

$30 \mid 2010$

Théorie : état des lieux

\title{
Les livres, les textes et la critique
}

\section{Hans Walter Gabler}

Traducteur : Sylvain Belluc

\section{(2) OpenEdition}

Journals

\section{Édition électronique}

URL : https://journals.openedition.org/genesis/109

DOI : $10.4000 /$ genesis. 109

ISSN : 2268-1590

\section{Éditeur :}

Presses universitaires de Paris Sorbonne (PUPS), Société internationale de génétique artistique littéraire et scientifique (SIGALES)

\section{Édition imprimée}

Date de publication : 20 juin 2010

Pagination : 41-42

ISBN : 978-2-84050-697-3

ISSN : 1167-5101

\section{Référence électronique}

Hans Walter Gabler, «Les livres, les textes et la critique », Genesis [En ligne], 30 | 2010, mis en ligne le 30 mai 2012, consulté le 30 mars 2023. URL : http://journals.openedition.org/genesis/109 ; DOI :

https://doi.org/10.4000/genesis. 109 


\title{
Les livres, les textes et la critique
}

\author{
Hans Walter Gabler
}

$\mathrm{L}$ es critiques textuelles allemande et anglo-américaine modernes ont vu le jour vers la fin du XIXe siècle. Elles sont nées - bien que chacune se soit développée à sa façon - d'un terreau commun de critique textuelle (ou philologie) appliquée aux transmissions classiques et médiévales. Une raison commune de leur émergence en tant que disciplines distinctes se trouve dans l'esprit du temps, et dans son intérêt pour la nation et la littérature nationale. Dans le même temps, cependant, c'est précisément la diversité des histoires nationales et des cultures et la prolifération des littératures nationales, qui ont donné naissance à des critiques textuelles aux profils distincts. À mesure que le $\mathrm{xx}^{\mathrm{e}}$ siècle avançait, ce qui a fini par les distinguer de la manière la plus nette, c'est leurs méthodologies considérablement différentes.

Dans le monde anglophone, c'est l'étude du livre qui a ouvert la voie à un mode de critique textuelle novateur aspirant à établir une assise scientifique. La bibliographie comme méthode de critique textuelle a pris une telle ampleur qu'au plus fort de l'engouement bibliographique exclusif des années soixante, les termes «bibliographie » et « critique textuelle » étaient devenus pratiquement interchangeables. En dehors de la sphère anglo-américaine, la «voie bibliographique »n'a jamais été adoptée sans quelque réticence, bien qu'elle fût prisée en tant que complément face aux complexités méthodologiques de la discipline. Dans les milieux de la recherche textuelle britannique et (plus encore) américaine, il devint de plus en plus difficile de réduire la critique textuelle à des considérations purement bibliographiques à mesure que prenaient forme les théories contemporaines du texte, ainsi que celles de la créativité et de l'écriture, et qu'on comprenait de mieux en mieux les modes de production et de transmission textuelles modernes. Malgré cela, tandis que le discours théorique des dernières décennies du siècle précédent s'efforçait d'élargir les perspectives, la bibliographie, même quand on la rejetait, continuait à exercer son influence. C'est ce qu'on peut observer dans les travaux actuels sur l'histoire du livre.
Pour les études savantes du livre, la synonymie d'autrefois semble aujourd'hui porter de remarquables fruits. Avant d'être rattachée à la critique textuelle, la bibliographie se limitait le plus souvent à une pratique consistant à répertorier les livres avant tout comme objets matériels, et sous forme de classification, de taxinomie et d'énumération. Dans l'histoire du livre telle qu'on l'écrit de nos jours, les livres sont perçus bien davantage dans leur relation aux textes qu'ils renferment et incarnent matériellement. Pour cette raison, il est possible d'affirmer que la recherche textuelle a contribué à ce que l'« Histoire du livre » soit reconnue comme un domaine essentiel des cultural studies.

Mais il doit être admis, réciproquement, que l'étude du livre ne concerne qu'indirectement la critique textuelle et la science éditoriale. Bien entendu, il ne fait aucun doute que les apports de la bibliographie textuelle et analytique à l'étude des textes sont bien établis. Ceci est d'autant plus vrai que la vision plus globale de la sociologie des textes et de la transmission textuelle qui a découlé de ces apports représente une intégration particulièrement poussée de la bibliographie au sein de la critique textuelle, même si elle ne représente que l'une des nombreuses méthodes qui lui apportent leur concours.

Ce qui est par ailleurs au moins aussi important, c'est que le $\mathrm{XX}^{\mathrm{e}}$ siècle a vu naître, dans les domaines de la critique textuelle et de l'édition, des changements d'orientation autres que ceux concernant le livre ou la bibliographie. La recherche éditoriale allemande, par exemple, choisit d'historiciser les textes plutôt que les livres. Ceci a permis d'élargir le nombre d'options offertes à la critique textuelle et à l'édition, de la historisch-kritische Edition, d'inspiration positiviste, à la Handschriftenedition, d'orientation génétique, en passant par la Fassungsedition, historiquement déterminée. Cette progression implique, me semble-t-il, une mutation : on est passé d'une historicisation des transmissions à une prise en compte de la genèse des textes. Cette prise en compte a pour effet, à son tour, de faire redécouvrir la fonction critique de la critique textuelle. 
Nous sommes par là même mis au défi de redécouvrir les fondations de la critique tout court. Au niveau fondamental de la théorie, ce défi, il faut le reconnaitre, n'est pas encore tout à fait relevé. À un niveau pragmatique, en revanche, on peut déjà discerner de nouvelles perspectives. Elles concernent en particulier la reconnaissance du manuscrit d'auteur moderne en tant qu'objet d'analyse, d'interprétation et d'édition à part entière, et à part entière seulement si l'on accepte de voir en lui quelque chose de bien plus riche et plus complexe que les traces du texte dont il est le témoin. À juste titre, nous considérons avec la plus grande attention les enseignements que la critique génétique nous dispense à cet égard, ce mouvement ayant ouvert la voie en accordant toute l'attention qu'il mérite à l'héritage matériel et culturel des manuscrits d'auteur. Les principes de la critique génétique, pendant ce temps, gagnent également du terrain dans le monde anglo-américain, où le concept dit de "fluidité textuelle » se répand et est à l'origine à la fois de nouveaux types d'éditions savantes et de discours interprétatifs d'un genre nouveau, à partir d'un travail critique sur les textes et les processus textuels.

À ceci, je voudrais ajouter que, lorsque ce type de discours critique orienté vers les processus porte sur les brouillons, il opère - fait exceptionnel - à deux niveaux. $\grave{A}$ un premier niveau, commenter les manuscrits et leur texte est un acte de critique performatif dans le sens où l'on entend d'ordinaire le terme " critique », c'est-à-dire un acte en réaction au texte en train d'être écrit. À un second niveau, cependant, il est possible de développer, à partir des brouillons, un discours critique séparé visant à interpréter de manière plausible l'écriture du manuscrit (par opposition à son texte) afin d'y voir des indications, ou des indices, des processus de pensée ou des actes décisionnels derrière le processus d'écriture. C'est pourquoi étudier les brouillons, c'est discerner de féconds actes de lecture inscrits dans les actes d'écriture. On peut les retrouver dans les brouillons ou bien seulement aux moments de révision des manuscrits ultérieurs. Ils sont absents des copies au net et des textes définitifs imprimés, qui, situés au-delà des processus d'écriture, préservent le texte uniquement comme le résultat et le produit de ces processus.

La critique textuelle qui, en se fondant à la fois sur le texte et l'écriture, prend appui sur des documents, renferme, me semble-t-il, un important potentiel d'innovation pour la critique. Elle devrait également servir à renouveler les concepts et les pratiques d'édition. En revanche, et pour revenir une fois de plus à la question de l'histoire du livre, le regain d'intérêt actuel pour cette histoire à partir de prémisses (pas toujours explicites ou clairement définies) se rapportant à la science des textes ne me semble ni innocemment tourné vers l'avenir, ni propice à l'intégration d'autres disciplines. La question à poser est plutôt celleci : les travaux sur l'histoire du livre, entrepris par les spécialistes de la science des textes avec un enthousiasme irréfléchi, ne seraient-ils pas un signe de régression? Je soupçonne que, puisque la « voie bibliographique » n'a plus cours en critique textuelle, la bibliographie est en train de redevenir actuellement de la « simple » bibliographie, sans plus se préoccuper des textes et de leur édition en fonction de prémisses bibliographiques. Si c'est le cas, que ce fait soit reconnu comme tel et, si on le souhaite, pratiqué avec profit. Mais que l'on reconnaisse également que cela ne devrait pas se faire au détriment de la critique textuelle et de l'édition. La théorisation de la science éditoriale, qu'elle soit pratiquée en son sein ou de l'extérieur, ralentit quelque peu, mais elle n'a pas encore donné naissance à une pratique éditoriale fondamentalement neuve, encore moins à des paradigmes nouveaux et stables pour les éditions savantes. Et pourtant, si désireux que je sois de voir pratiquer une historicisation sophistiquée du livre (du passé), je le serais au moins autant de voir naître une édition savante du futur et pour le futur et plus encore de voir la critique textuelle affirmer son statut de branche essentielle de la critique littéraire.

Traduit de l'anglais par Sylvain Belluc

Hans Walter Gabler a été professeur de littérature anglaise à l'université de Munich et il y a conduit de 1996 à 2002 un séminaire interdisciplinaire de troisième cycle sur « La critique textuelle comme fondement et comme méthode pour les disciplines historiques ». Il a été le responsable des éditions critiques de Ulysses (1984-1986), de A Portrait of the Artist as a Young Man (1993) et de Dubliners (1993) de James Joyce. Il s'intéresse actuellement aux processus d'écriture tels qu'ils apparaissent dans les manuscrits d'écrivains, à leur interprétation critique et à leur représentation sous forme électronique.

Hans Walter Gabler, Gabler@anglistik.uni-muenchen.de 